3. Wardrop CAJ, Holl and BM, Veale KEA, Jones JG, Gray OP: Nonphysiologic anemia of prematurity. Arch Dis Child .53: 855, 1978.

\section{Neonatal Polycythemia and Hyperviscosity Syndrome}

\section{VIRGINIA D. BLACK}

\section{Departments of Pediatrics, Wayne State University, Hutzel Hospital and Children's Hospital of Michigan, Detroit, Michigan U.S.A.}

Infant hematocrit varies with both gestational age and postnatal age. It has been assumed that the rise in hematorit which occurs from first to third trimester is an adaptation to increase oxygen needs and the relative intrauterine hypoxia. Three decades ago newborns with markedly elevated hematocrits and serlous cardiorespi ratory distress were described. Often the symptomatic infant was the recipient of a twin-to-twin transfusion. These early case reports are the first documented cases of what is now described as neonatal polycythemia.

In 1966, Baum (1) Identified an elevated viscosity in blood from infants who were polycythemic. This elevation in viscosity was belfeved to decrease peripheral blood flow and hence mlght explain the pathology assoclated with neonatal polycythemla. A new condition, neonatal hyperviscosity, was described.

It is common but Inaccurate to equate neonatal polycythemia and neonatal hyperviscosity. The definitlons of each are specific and, although overlapping, do not exactly define the same population.

Neonatal polycythemla is most commonly descrlbed as a venous hematocrit of $65 \%$. Levels as low as $60 \%$ or as high as $70 \%$ have also been suggested. Variations in sample site or postnatal age compound the difficulty in defining neonatal polycythemla. Linderkamp (3) and Gatt I (2) have demonstrated the varlability between capillary and venous hematocrit values. Shohat (4) was able to demonstrate dynamic changes in both venous hematocrit and viscosity measurements. By two hours of age there is a linear correlation between cord blood hematocrlt and venous hematocrit. Thus, cord blood hematocrit may be a reliable technique for initlal screening. However, this technique may miss infants who become polycythemic as a result of acute placental transfusion.

Neonatal hyperviscosity refers to a characteristic of blood which Increases with henatocrit. Several other factors al so contribute to the viscosity of whole blood. Among these are large plasma proteins and stiffness of the red blood cell membrane. Newborm plasma has a lower viscosity compared to adult plasma since the concentration of plasma proteins is lower in the newborn. Standards for viscosity measurements are available from several authors. Hyperviscosity has been defined as a value more than two standard deviations from mean cord viscosity. Whole blood viscosity is dependent on the shear rate studied. Viscosity measurements are therefore given over several shear rates. Use of the viscosity standards avallable suggest that 2-6\% of newborn infants have hyperviscosity. Whl le hematocrit and viscosity are closely correlated the contribution of other factors to viscosity nake it impossible to equate the two conditions. At the higher henatocrit levels all polycythemic infants are hyperviscous. Some infants without polycythenla have abnomal whole blood viscosity. Some polycythemic infants are hyperviscous. In the remainder of this review the clinlcal signs and outcome assoclated with the two conditions will be descussed.

Infants with polycythemla and hyperviscosity often present with cardioresplratory signs which mimic cyanotic concenltal heart disease. Tachypnea, tachycardia, cyanosis, and apnea have all been described as well as pulmonary hypertension.

Central nervous system signs are common including the non specific findings of irritability, rapid state changes, vasomotor instability, lethargy, poor tone, and poor feeding. Early reports of polycythemic infants frequently reported seizures. Prospective screening of infants has identified few with neonatal seizures. Obvious central nervous system hemorrhage is also un cammon.

More recently there has become Increasing evidence that polycythemla/hyperviscosity is a frequent cause of necrotlzing enterocolitis (NEC) when it appears in the term infant. Polycythemic infants undergolng partial plasma exchange with fresh cythemic Infants undergolng partial plasma exchange with fresh frozen plasma seem particularly vulnerable to developing a specalso been seen in many of these infants with a range of gastrointestinal signs.

Renal fallure, thrombocytopenia, and various other hematologic findings have also been reported. Hypoglycemia is cormonly associated with polycythemia and may be of greater magnitude than mates whole blood glucose. Peripheral blood flow may be severely mates whole blood glucose. Peripheral blood flow may be

compromised and has improved following plasmaphoresis.
Outcome following neonatal polycythemla/hyperviscosity is largely dependent upon the method used to identify the subjects. Serlously compromlsed infants as orlginally descrlbed are not the rule. Most infants have symptoms that are less obvious. The most symptomatic infants in early studies had developmental delays and neurologlc handicaps. Prospective screening identifles a group of infants for whom the outcome remains controversial. Several auinfants for whom the outcome remains controversial. Several auour studies suggest that there are clear differences between polycythemic Infants and a comparison group at two years of age.
Partial plasma exchange appears to Improve sensory outcome. All studies of prospectively identified polycythemic infants suggest that mental development is not significantly affected.

\section{REFERENCES}

1. Baum RS, 1966, Viscous forces in neonatal polycythemia. J Pedlatr 69: 975

2. Gattl RA, 1967, Hematocrit values for caplllary blood in the newborn infant. J Pedlatr 70: 117

3. Linderkamp 0, Versmold HT, Strohhacker 1, et al, 1977, Capillary-venous hematocrit differences in newborn infants. Eur J Pediatr 127: 9

4. Shohat M, Merlob P, Reisner SH, 1984, Neonatal polycythemia: 1. Early diagnosis' and incidence relating to time of sampling. Pediatrics 73: 7

\section{Oxygen Radicals and Tissue Damage in the Perinatal Period}

\section{OLA D. SAUGSTAD}

\section{Department of Pediatrics, The National Hospital, Oslo, Norway}

Posthypoxic tissue damage could be caused by oxygen free radicals generated by hypoxanthine $(\mathrm{Hx})$-xanthine oxidase $\left(\mathrm{XO}_{0}\right)$ system. $\mathrm{Hx}$ which is accumulated in hypoxia is washed out into the circulation in the posthypoxic reperfusion period. If $X O$ is present the superoxide radical, $\mathrm{O}_{2}^{-}$, is formed: $\mathrm{Hx}+\mathrm{O}_{2} \underset{\mathrm{xO}^{-}}{\longrightarrow}$ Urate $+\mathrm{O}_{2}^{-}$.

We have suggested that this mechanism is responsible, at least partly, for several conditions in peri/neonatal medicine such as bronchopulmonary dysplasia, necrotizing enterocolitis and retrolental fibroplasla. If this hypothesis is correct we are dealing with a cormon pathogenetic mechanism leading to one disease affecting different organs.

To explore this hypothesis we have mainly studled the lung. In sumary we have found: 1) The combination of 100 percent oxygen and int ravenous oxygen given $48 \mathrm{~h}$ damage the rat lung. The typical changes found are hemorrhage and edema. Furthermore, in spite of a nortmal lung surfactant profile the surfactant function was abolished indicating that surfactant was inactivated elther by peroxidation or by the induction of surfactant Inhibitor. 2) Xo applied into the al rways of guinea pigs acute?y induced lung hemorrhage and edema. The lung compliance was dramatically reduced. One single application of $X 0$ induced long term effects on the lings as well. After 2 days thickening of alveolar septae and proliferation of type 2 cells were observed. Furthermore, there was an increased number of alveolar macrophages. 7 and 14 days afterwards the histological changes seemed to have normalized, lung compliance, however, was stil signlficantly lowered. 3) Superoxide dismutase given together with XO protected against Xo.

The experimental data show that the hypoxanthine-xanthine ox:dase system potently destroys the lungs under experimental conditions.

\section{Red Cell Aggregation in the Neonate}

\section{MICHAEL RAMPLING}

\section{Department of Physiology and Biophysics, St. Mary's Hospital Medical School, London W2, UK}

The viscometric characteristics of neonatal blood show distinct differences from those of adults $(1,2)$; in particular it is found that at a given haematocrit neonatal blood generally exhibits lower viscosity at all shear rates. In part this is due to the lower plasma viscosity that is also found in the neonate. The effect of this plasma difference can be removed by calculating relative viscosity, and when this is done the high shear rate $\left(>5 \mathrm{~s}^{-1}\right)$ values of the two types of blood become similar but the low shear rate difference persists.

Since it is usually accepted that the elevated low shear rate viscosity of adult blocd is due to rouleaux formation, it is to be expected that the latter will be reduced in the neonate and this has been confirmed experimentally.

The cause of the reduced aggregation must lie in the red cell or in the plasma or both. However plasma exchange experiments indicate that adult and neonatal cells aggregate similarly when suspended in adult plasma, while both show reduced aggregation in neonatal plasma (2). Of the aggregating agents in adult blood fibrinogen is by far the most important, so experiments have been performed in which both cell types were suspended in solutions of purified adult fibrinogen. Again they showed similar degrees of rouleaux formation. The conclusion is therefore that the reduced aggregation found in neonata? blood is not due to cellular differences, but rather to variations in the composition of the plasma. 